\title{
Influence of ampoule rotation on three-dimensional convection and segregation in Bridgman crystal growth under imperfect growth conditions
}

\author{
C.W. Lan*, M.C. Liang, J.H. Chian \\ Chemical Engineering Department, National Taiwan University, Taipei 10617, Taiwan, ROC
}

Received 17 August 1999; accepted 10 December 1999

Communicated by D.T.J. Hurle

\begin{abstract}
Three-dimensional (3D) convection and segregation due to imperfect growth conditions, such as ampoule tilting and asymmetric heating, are common problems in vertical Bridgman crystal growth. How to suppress the 3D effects has been an important task for better growth control. To investigate the possibility of using steady ampoule rotation to damp the 3D flows, numerical simulation is conducted. It is found that the low-speed rotation (e.g., $10 \mathrm{RPM}$ ) can reduce significantly the 3D flows, but may result in larger radial segregation due to less dopant mixing, as well as rotational growth and melting. For weaker convection, which corresponds to a low-thermal-gradient or reduced-gravity growth, ampoule rotation is particularly effective. This is especially true in space, where the 3D flows and segregation induced by an arbitrary residual gravity can be significantly suppressed by only several RPM of ampoule rotation leading to a nearly diffusion-controlled growth. However, if the growth interface is not axisymmetric, which is often caused by an asymmetric heating, rotational segregation can be quite severe, even without the buoyancy convection. (C) 2000 Elsevier Science B.V. All rights reserved.
\end{abstract}

PACS: 44.25. + f; 47.27.Te; 81.10.Fq; 02.60.Cb; 02.70.Fj

Keywords: 3D simulation; Bridgman method; Rotation; Interface; Buoyancy convection; Segregation

\section{Introduction}

The Bridgman technique is a simple and useful process and has been widely used in growing highquality single crystals (e.g., Refs. [1-4]). However, the heat flow and segregation in the process strong-

\footnotetext{
*Corresponding author. Tel.: + 886-2-2363-3917; fax: + 886-2-2363-3917.

E-mail address: lan@ruby.che.ntu.edu.tw (C.W. Lan).
}

ly depend on ampoule orientation and heating uniformity. To minimize the unstable flow and convection, the ampoule is usually aligned with the gravity orientation and the melt sits upon the growth interface, and this the so-called vertical Bridgman (VB) configuration. However, perfect alignment and uniform heating are hard to obtain in practice. As indicated by the three-dimensional (3D) simulations of Liang and Lan [5] and Xiao et al. [6], a slightly tilted ampoule (less than $2^{\circ}$ ) or a small heating nonuniformity $\left(2^{\circ} \mathrm{C}\right.$ in the 
azimuthal direction) can induce significant 3D flows and thus 3D segregation. The situation becomes worse as the buoyancy force increases. On the other hand, the enhanced 3D flows increases the dopant mixing and may reduce segregation [6], but the dopant uniformity is not axisymmetric. Even in microgravity condition, the situation may not be improved much because the gravity orientation is never fixed during the whole growth period. The weak flow induced by the residual gravity sometimes induces an even severe segregation [5]. Therefore, how to better control or suppress the 3D effects is highly desirable for $\mathrm{VB}$ crystal growth. In fact, the situation can become even more complicated as the solutal effect is taken into account.

The most effective way to suppress the $3 \mathrm{D}$ effects is to damp the convection sufficiently to reach the diffusion-controlled limit. Indeed, the most wellknown approach is the use of magnetic damping (e.g., Refs. [7-9]). The flow intensity $\left(\psi_{\max }\right)$ decreases with the increasing magnetic field strength $\left(B\right.$ or in terms of Hartmann number Ha); $\psi_{\max } \sim$ $\mathrm{Ha}^{-2}$ [7]. However, the hardware requirement to provide a sufficient magnetic damping (usually in the order of $0.5 \mathrm{~T}$ ) is costly. An alternative way to suppress the flow, maybe other mechanisms like vibration [10] as well, is the use of steady ampoule rotation. As discussed by Lan and Chian [11,12], as well as Yackel et al. [13], a moderate rotation rate may affect the buoyancy flow significantly for the axisymmetric configuration. In addition, its damping effect is similar to that due to the magnetic field but less effective; $\psi_{\max } \sim \Omega^{-1}$ [14], where $\Omega$ is the rotation speed. For the situation that the buoyancy flow is weak, the ampoule rotation may be an effective tool for the flow control. More importantly, as the growth is subjected to the imperfect growth conditions as mentioned previously, the $3 \mathrm{D}$ flows may also be suppressed. However, the influence of ampoule rotation on the 3D flows and segregation has not been explored. Furthermore, as the growth front is not axisymmetric, which may be caused by the convection or asymmetric heating, the rotational effects are particularly interesting. The rotational growth and melting, which is believed to be the source of rotational striations, can lead to severe segregation and constitutional super- cooling. Again, no such a numerical study has been reported. Using a centrifuge may also be useful in the flow control [15], but the convection is not suppressed and the 3D effect is introduced.

In the present study, a 3D numerical simulation is conducted to investigate the effects of ampoule rotation on the 3D flows and segregation under imperfect growth conditions. The effects of both gravity orientation (ampoule tilting) and asymmetric heating are considered. To further illustrate the feasibility of using ampoule rotation for flow damping, different convection intensities are considered and the reduced gravity situation is discussed. In the next section, the model and its numerical simulation are briefly described. Section 3 is devoted to the results and discussion, followed by conclusions and comments in Section 4.

\section{Model description and numerical solution}

A generic Bridgman crystal growth system is illustrated in Fig. 1a. Since axisymmetry is no longer assumed here, the system is described by a Cartesian coordinate $(x, y, z)$. The asymmetry could be introduced by the tilted ampoule, which is represented by the tilt angle $\gamma$, or the nonuniform ambient thermal profile $T_{\mathrm{a}}(x, y, z)$. In Fig. 1a, each region is characterized by a set of physical properties. Since our major interest in this paper is the effects of rotation on the flows, to simplify the discussion, a pseudo-steady-state growth is further assumed, where the ampoule pulling speed $U_{\text {amp }}$ is set to be the axial growth rate. The flow, temperature, and dopant fields, as well as the melt/crystal interface, are also represented in the Cartesian coordinate $(x, y, z)$. The ampoule is rotated around the growth axis at a constant rotation speed $\Omega$. Because the coordinate we have chosen is a fixed frame, it is not possible to describe the steady-state dopant field in the crystal. Therefore, the dopant segregation calculated in the crystal is only a snap shot of the growth, not a real distribution in the crystal. Accordingly, the dopant boundary condition at the growth front due to the crystal side is only an approximation. Furthermore, it is also assumed that the dopant concentration is very low so that both flow and liquidus temperature are not 


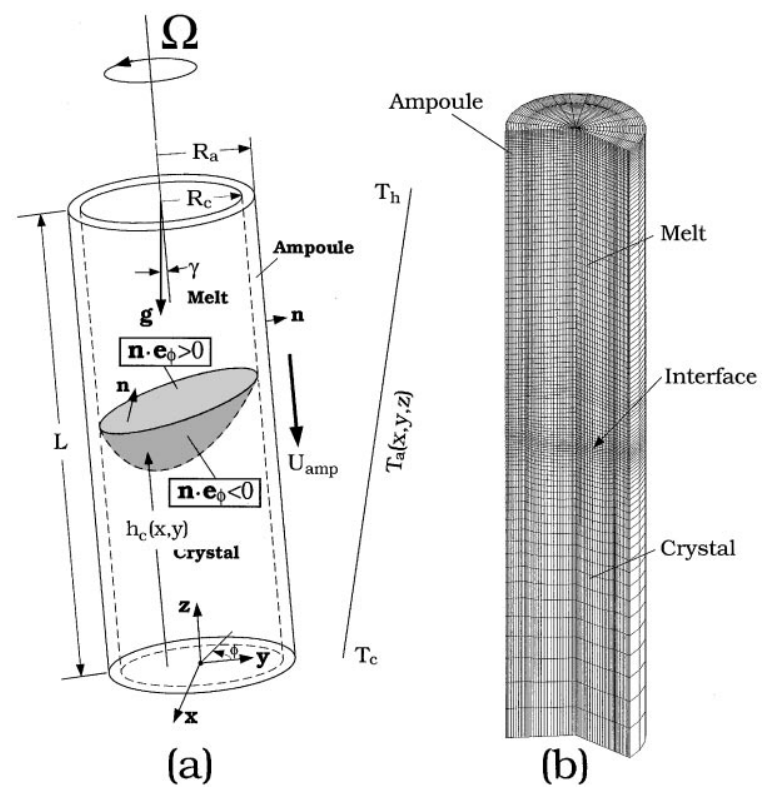

Fig. 1. (a) Schematic sketch of Bridgman crystal growth with ampoule rotation; (b) a portion of a sample mesh for calculation.

affected. Therefore, the calculated flow field is not affected by the dopant distribution.

The dimensionless variables are defined by scaling length with the ampoule length $L$, velocity with $\alpha_{\mathrm{m}} / L$, pressure with $\rho_{\mathrm{m}} \alpha_{\mathrm{m}}^{2} / L^{2}$, and dopant concentration with its average concentration $C_{0}$ in the crystal, where $\alpha_{\mathrm{m}}$ is the thermal diffusivity and $\rho_{\mathrm{m}}$ the melt density. The dimensionless temperature $(\theta)$ is defined as

$\theta(x, y, z)=\left[T(x, y, z)-T_{\mathrm{c}}\right] /\left(T_{\mathrm{h}}-T_{\mathrm{c}}\right)$,

where $T_{\mathrm{h}}$ and $T_{\mathrm{c}}$ are the top (hot) and bottom (cold) temperatures, as shown in Fig. 1a. The pseudo-steady-state governing equations describing convection and heat and dopant transport in the melt (m) are as follows:

$\nabla \cdot v=0$,

$\boldsymbol{v} \cdot \nabla \boldsymbol{v}=-\nabla P+\operatorname{Pr} \nabla^{2} \boldsymbol{v}-\operatorname{PrRa}_{\mathrm{T}}\left(\theta-\theta_{\mathrm{m}}\right) \boldsymbol{e}_{\mathrm{g}}$,

$\boldsymbol{v} \cdot \nabla \theta=\nabla^{2} \theta$,

$\boldsymbol{v} \cdot \nabla C=\frac{\operatorname{Pr}}{\mathrm{Sc}} \nabla^{2} C$, where $\boldsymbol{v}, P$, and $C$ are the dimensionless velocity, pressure, and dopant concentration, respectively. $\operatorname{Pr}$ is the Prandtl number $\left(\operatorname{Pr} \equiv v_{\mathrm{m}} / \alpha_{\mathrm{m}}\right)$, Sc the Schmidt number $\left(\mathrm{Sc} \equiv v_{\mathrm{m}} / D\right)$, and $\theta_{\mathrm{m}}$ the melting temperature, which also serves as a reference temperature; $v_{\mathrm{m}}$ is the kinematic viscosity and $D$ the dopant diffusivity in the melt. The thermal Rayleigh number $\mathrm{Ra}_{\mathrm{T}}$ in the source term of the momentum equation is defined as follows:

$\mathrm{Ra}_{\mathrm{T}} \equiv \frac{g \beta_{\mathrm{T}} \Delta T L^{3}}{\alpha_{\mathrm{m}} v_{\mathrm{m}}}$

where $g$ is the gravitational acceleration, $\beta_{\mathrm{T}}$ the thermal expansion coefficients, and $\Delta T=$ $T_{\mathrm{h}}-T_{\mathrm{c}}$. Also, if one represents the equation of motion in terms of a rotating frame, the Coriolis force due to steady rotation becomes obvious being $2 \operatorname{PrTa}^{1 / 2} \boldsymbol{e}_{\phi} \times \boldsymbol{v}$ and $\mathrm{Ta} \equiv \Omega^{2} R_{\mathrm{c}}^{4} / \nu_{\mathrm{m}}^{2}$ is the Taylor number. This term is similar to the Lorentz body force for magnetic damping [14]. The gravity direction $\boldsymbol{e}_{\mathrm{g}}$ can be decomposed into the Cartesian components $\left(\boldsymbol{e}_{x}, \boldsymbol{e}_{y}, \boldsymbol{e}_{z}\right)$ :

$\boldsymbol{e}_{\mathrm{g}}=\cos (\alpha) \boldsymbol{e}_{x}-\cos (\beta) \boldsymbol{e}_{y}-\cos (\gamma) \boldsymbol{e}_{z}$,

where the $\alpha, \beta$, and $\gamma$ are the angles between the gravity direction and the axes of $x, y$, and $z$, respectively. For a perfectly aligned ampoule, $\alpha=\beta=\pi / 2$, and $\gamma=0$. In the present study, nonaxisymmetry is introduced only by tilting the ampoule in the $y-z$ plane, i.e., $\alpha=\pi / 2$.

In the crystal (c) and the ampoule (a), only heat transfer needs to be considered:

$\left(-\mathrm{Pe}_{i} \boldsymbol{e}_{z}+r \mathrm{Pe}_{i}^{\Omega} \boldsymbol{e}_{\phi}\right) \cdot \nabla \theta=\kappa_{i} \nabla^{2} \theta, \quad(i=\mathrm{c}, \mathrm{a})$,

where $\mathrm{Pe}_{i} \equiv \rho_{i} C p_{i} U_{\mathrm{amp}} L / k_{\mathrm{m}}$ is the translational Peclect number, $\mathrm{Pe}_{i}^{\Omega} \equiv \rho_{i} C p_{i} \Omega L^{2} / k_{\mathrm{m}}$ the rotational Peclect number, $\boldsymbol{e}_{\phi}$ the angular unit vector, $r$ the dimensionless radial coordinate, and $\kappa_{i} \equiv k_{i} / k_{\mathrm{m}}$ the dimensionless thermal conductivity of crystal or ampoule; $k_{\mathrm{m}}$ is the thermal conductivity of the melt. Also, $\rho_{i}, C p_{i}$, and $k_{i}$ are the density, specific heat, and thermal conductivity of the phase $i(i=\mathrm{c}$ or a), respectively.

The no-slip condition is used for the melt velocity on solid boundaries:

$\boldsymbol{v}=\gamma_{\mathrm{c}}\left(-v_{\mathrm{amp}} \boldsymbol{e}_{z}+r \Omega^{*} \boldsymbol{e}_{\phi}\right)$, 
where $\gamma_{\mathrm{c}} \equiv \rho_{\mathrm{c}} / \rho_{\mathrm{m}}$ and $v_{\mathrm{amp}}$ is the dimensionless ampoule pulling speed and $\Omega^{*}$ the dimensionless angular rotation speed; $\gamma_{\mathrm{c}}$ is assumed to be one in this study. Since the pseudo-steady-state is assumed, the upper open boundary is considered as an artificial boundary [5], and its velocity boundary condition is the same. The thermal and solutal boundary conditions at the melt/solid interfaces are set by the heat and mass flux balances. For example, at the growth front:

$$
\begin{aligned}
\boldsymbol{n} \cdot & \left.\nabla \theta\right|_{\mathrm{m}}-\left.\boldsymbol{n} \cdot \kappa_{\mathrm{c}} \nabla \theta\right|_{\mathrm{c}} \\
& +\gamma_{\mathrm{c}}\left(\mathrm{Pe}_{\mathrm{m}} \boldsymbol{e}_{z}-r \mathrm{Pe}_{\mathrm{m}}^{\Omega} \boldsymbol{e}_{\phi}\right) \mathrm{St} \cdot \boldsymbol{n}=0 \\
- & \left.\boldsymbol{n} \cdot \nabla C\right|_{\mathrm{m}}-\gamma_{\mathrm{c}}\left(\mathrm{Pe}_{\mathrm{m}} \boldsymbol{e}_{z}-r \mathrm{Pe}_{\mathrm{m}}^{\Omega} \boldsymbol{e}_{\phi}\right) \\
& \times \frac{\mathrm{Sc}}{\operatorname{Pr}}(1-K) C \cdot \boldsymbol{n}=0
\end{aligned}
$$

where $\boldsymbol{n}$ is the unit normal vector at the growth interface pointing to the melt. The Stefan number $\mathrm{St} \equiv \Delta H /\left(C p_{\mathrm{m}} \Delta T\right)$ scales the heat of fusion $(\Delta H)$ released during solidification to the sensible heat in the melt. The equilibrium segregation coefficient $K$ of the solute is according to the phase diagram; $K \equiv C_{\mathrm{c}} / C$ at the growth interface, where $C_{\mathrm{c}}$ is the dopant concentration in the crystal.

It should be pointed out that if the interface is not axisymmetric, $\left(\boldsymbol{e}_{\phi} \cdot \boldsymbol{n}\right)$ is not zero. As a result, for a steady-state growth, continuous melting and growth continue even the ampoule is not pulled downward. Accordingly, the heat of fusion also increases the interface shift (an isotherm surface) along the azimuthal direction. Furthermore, for dopant segregation, the growth $\left(\boldsymbol{e}_{\phi} \cdot \boldsymbol{n}<0\right)$ increases the concentration in the growth front, while the melting $\left(\boldsymbol{e}_{\phi} \cdot \boldsymbol{n}>0\right)$ decreases the concentration. Nevertheless, as mentioned previously, this is not always realistic because we have assumed that at the growth front an equilibrium exists, i.e., $C_{\mathrm{c}} / C=K$, even for melting. Therefore, Eq. (10) provides only an approximation. For a detailed simulation, time-dependent calculation including the solid-state diffusion should be included. However, at the present stage, it is still too costly to do so. Another approach assuming that the solid is swallowed by the melt during melting can be used as the feed front boundary condition used in the zone-melting modeling [16], but the result is sim- ilar to that of Eq. (10). As it will be discussed shortly, for asymmetric heating, severe segregation due to this effect is observed, and this is similar to the cause of the rotational striations in Czochralski growth [17]. Furthermore, in our steady-state calculations, since the fixed-frame is chosen, except at the growth interface, it is also not possible to describe the dopant field in the crystal. Indeed, if the solid-state diffusion is neglected, a spiral dopant distribution is expected for the cases with 3D flows. Also, the temperature at the melt/solid interface is assumed to be the equilibrium liquidus temperature of the material.

Temperature at the top and bottom surfaces is set to be the furnace temperature there. With the pseudo-steady-state assumption, an artificial boundary condition is used at the upper boundary for the consistence of the overall dopant balance [5]:

$$
-\left.\boldsymbol{n} \cdot \nabla C\right|_{\mathrm{m}}=\gamma_{\mathrm{c}} \frac{\operatorname{Pe}_{\mathrm{m}} \mathrm{Sc}}{\operatorname{Pr}}(C-1)\left(\boldsymbol{n} \cdot \boldsymbol{e}_{z}\right) .
$$

The heat exchange between the ampoule and the furnace is by both radiation and convection according to the energy balance along the ampoule surface,

$$
-\left.\boldsymbol{n} \cdot \kappa_{\mathrm{a}} \nabla \theta\right|_{\mathrm{a}}=\operatorname{Bi}\left(\theta-\theta_{\mathrm{a}}\right)+\operatorname{Rad}\left(\theta^{4}-\theta_{\mathrm{a}}^{4}\right),
$$

where $\boldsymbol{n}$ is the unit normal vector on the ampoule surface pointing outwards, $\mathrm{Bi} \equiv h L / k_{\mathrm{m}}$ the Biot number, and $\operatorname{Rad} \equiv \sigma \varepsilon_{\mathrm{a}} \Delta T^{3} L / k_{\mathrm{m}}$ the Radiation number; $\sigma$ is the Stefan Boltzmann constant, while $\varepsilon_{\mathrm{a}}$ is the surface emissivity of the ampoule. For simplicity, the effective furnace temperature $\theta_{\mathrm{a}}$ is assumed to be

$\theta_{\mathrm{a}}(x, y, z)=z+\theta_{\mathrm{d}}(x, y, z)$,

where $\theta_{\mathrm{d}}(x, y, z)$ is a deviation temperature to describe thermal nonuniformity. A Gaussian distribution is used for $\theta_{\mathrm{d}}$ :

$\theta_{\mathrm{d}}(x, y, z)=\Delta \theta_{\mathrm{d}} \exp \left[-\left[(z-0.5) / a^{*}\right]^{2}\right]$

and

$\Delta \theta_{\mathrm{d}}=-\Delta \theta_{\mathrm{dp}} \cos (\phi)$,

where $\Delta \theta_{\mathrm{dp}}$ is the dimensionless peak (maximum) deviation temperature difference along the 
azimuthal angle $(\phi)$ and $a^{*}$ a dimensionless parameter for the width of the distribution. In the present study, the nonaxisymmetry for a perfectly aligned ampoule is introduced by a nonzero $\Delta \theta_{\mathrm{dp}}$ for nonuniform heating. The complications of computing view factors from the sample to the furnace are neglected here. Furthermore, during ampoule rotation, we also assume that the thermal field around the environment is not affected, and the rotation should be co-axis. Otherwise, the steadystate temperature distribution is not possible. Another way that may be more convenient for simulation is to use a rotation frame for the whole growth system, including the ampoule and the furnace. However, in the present study, we still stick with the fixed-frame approach, from the observer point of view, for simulation.

The above governing equations and their associated boundary conditions can only be solved numerically. We have developed an efficient finite volume method (FVM) scheme using the primitive variable formulation [18] and multigrid acceleration [19] for the free boundary problem. This approach is much more efficient and robust than the previous FVM/Newton's method [5]. A sample converged mesh for calculation is shown in Fig. $1 \mathrm{~b}$. As shown, finer grid spacing is placed near the interfaces to enhance the accuracy of calculation. Detailed description of the numerical method can be found in Refs. [18,19].

\section{Results and discussion}

For comparison purposes, we consider the gallium-doped germanium $(\mathrm{GaGe})$ growth in the Grenoble furnace investigated by Adornato and Brown [20] in this study. The same system was also studied by Lan and Chen [21] as a benchmark problem for comparing the performance of a $2 \mathrm{D}$ FVM (stream function-vorticity $(\psi-\omega)$ formulation) and a Galerkin finite element method (primitive variable formulation). The effects of ampoule tilting and nonuniform heating for $\mathrm{Ra}_{\mathrm{T}}=0-10^{6}$ were further studied by Liang and Lan [5] using a FVM/Newton's method. Therefore, in this paper, we will extend the previous calculations to a much higher $\operatorname{Ra}_{\mathrm{T}}\left(\mathrm{Ra}_{\mathrm{T}}=2.489 \times 10^{8}\right.$, which corresponds
Table 1

Physical properties and input parameters $[5,11]$

$\mathrm{GaGe}$

$\rho_{\mathrm{c}}=5.5 \mathrm{~g} \mathrm{~cm}^{-3}$

$\rho_{\mathrm{m}}=5.5 \mathrm{~g} \mathrm{~cm}^{-3}$

$T_{\mathrm{m}}=937.4^{\circ} \mathrm{C}$

$\Delta H=460 \mathrm{~J} \mathrm{~g}^{-1}$

$k_{\mathrm{c}}=0.17 \mathrm{~W} \mathrm{~cm}^{-1}{ }^{\circ} \mathrm{C}^{-1}$

$k_{\mathrm{m}}=0.39 \mathrm{~W} \mathrm{~cm}^{-1}{ }^{\circ} \mathrm{C}^{-1}$

$C p_{\mathrm{c}}=C p_{\mathrm{m}}=0.39 \mathrm{~J} \mathrm{~g}^{-1}{ }^{\circ} \mathrm{C}^{-1}$

$\mu=0.00715 \mathrm{~g} \mathrm{~cm}^{-1} \mathrm{~s}^{-1}$

$\beta_{T}=5 \times 10^{-4}{ }^{\circ} \mathrm{C}^{-1}$

$D=2.1 \times 10^{-4} \mathrm{~cm}^{2} \mathrm{~s}^{-1}$

$K=0.087$

Graphite (ampoule)

$\rho_{\mathrm{a}}=1.8 \mathrm{~g} \mathrm{~cm}^{-3}$

$k_{\mathrm{a}}=3.26 \mathrm{~W} \mathrm{~cm}^{-1}{ }^{\circ} \mathrm{C}^{-1}$

$C p_{\mathrm{a}}=1.814 \mathrm{~J} \mathrm{~g}^{-1}{ }^{\circ} \mathrm{C}^{-1}$

$\varepsilon_{\mathrm{a}}=0$

Other input parameters

$L=7 \mathrm{~cm}$

$R_{\mathrm{c}}=0.5 \mathrm{~cm}$

$R_{\mathrm{a}}=0.7 \mathrm{~cm}$

$T_{\mathrm{h}}=1112.4^{\circ} \mathrm{C}$

$T_{\mathrm{c}}=762.4^{\circ} \mathrm{C}$

$\Delta T=T_{\mathrm{h}}-T_{\mathrm{c}}=350^{\circ} \mathrm{C}$

$U_{\text {amp }}=4 \times 10^{-4} \mathrm{~cm} / \mathrm{s}$

$h=46.571 \mathrm{~W} \mathrm{~cm}^{-2}{ }^{\circ} \mathrm{C}^{-1}$

$a=1 \mathrm{~cm}$

$\gamma=0-1.5^{\circ}$

$\Delta T_{\mathrm{dp}}=0-1^{\circ} \mathrm{C}$

$\Omega=0-10 \mathrm{RPM}\left(\mathrm{Ta} \equiv \Omega^{2} R_{\mathrm{c}}^{4} / v_{\mathrm{m}}^{2}=0-4.0556 \times 10^{4}\right)$

$\mathrm{Ra}_{\mathrm{T}} \equiv g \beta_{\mathrm{T}} \Delta T L^{3} /\left(\alpha_{\mathrm{m}} v_{\mathrm{m}}\right)=0-2.489 \times 10^{8}$

to a normal gravity condition for $\Delta T=350^{\circ} \mathrm{C}$ ) using the newly developed numerical scheme [19]. Furthermore, we will focus on the study of ampoule rotation on the 3D flows and segregation induced by imperfect growth conditions for the VB crystal system. Since the dimensionless variables are used for illustration, the calculated results may also be useful for other semiconductor systems with similar flow structures and dimensionless numbers. For reference, the physical properties of $\mathrm{GaGe}$ and some input parameters used in this study are listed in Table 1. Again, the condition of $\mathrm{Rad}=0$ used in 
Ref. [5] is also adopted here for comparison. The mesh used in the following calculations is similar to Fig. 1b. It contains 84864 finite volumes; $22 \times 24 \times 72(r, \theta, z)$ finite volumes in the melt. This leads to 276482 nonlinear equations. All computations are performed in an HP/9000-C180 workstation, and one calculation takes about $2 \mathrm{~h}$ of $\mathrm{CPU}$ time depending on the initial guess.

Before the effects of ampoule rotation are discussed, some numerical tests are necessary to ensure the correctness of calculations. Besides the extensive numerical tests being performed before $[5,11,21]$, we further consider the comparison with the $2 \mathrm{D}$ results for $\mathrm{Ra}_{\mathrm{T}}=2.489 \times 10^{8}$ (normal gravity) and $\mathrm{Ra}_{\mathrm{T}}=1 \times 10^{6}$ (reduced gravity). As shown in Fig. 2a, for $\mathrm{Ra}_{\mathrm{T}}=2.489 \times 10^{8}$, the calculated flow pattern (on the left-hand side) is in good agreement with the previous 2D result (e.g., Fig. 3c in Ref. [11]); all the detailed flow structures are revealed. However, still some discrepancy exist for the dopant field, which is shown on the right-hand side of Fig. 2a, but it is not significant numerically. Overall speaking, the agreement is quite satisfactory; one may examine the maximum dopant concentration in the melt $\left(C_{\max }=12.981\right.$ versus 13.047 (2D)). In this case, the calculated flow and solute fields are still 2D, which can be further examined by the dopant concentration at the growth interface, shown at the bottom of Fig. 2a. The dopant concentration in the crystal phase, $C_{\mathrm{c}}$, is obtained by multiplying its corresponding melt concentration with the segregation coefficient $K$, i.e., $C_{\mathrm{c}}=K C$. The second comparison for $\mathrm{Ra}_{\mathrm{T}}=1 \times 10^{6}$ at $5 \mathrm{RPM}$ ampoule rotation (or $\mathrm{Ta}=1.0139 \times 10^{4}$ ) is shown in Fig. 2b, and as shown the agreement is very good due to the much weaker convection. Further mesh refinement can improve the agreement, but it seems to be unnecessary. For stronger convection considered in this study, although the accuracy of the dopant fields can be degraded slightly, the error in the maximum dopant concentration is still quite acceptable by using the mesh similar to Fig. $1 b$.

In the following discussion, we will start the discussion on the rotation effects for ampoule tilting first and then for the asymmetric heating. Conclusions and comments are then given in Section 4.

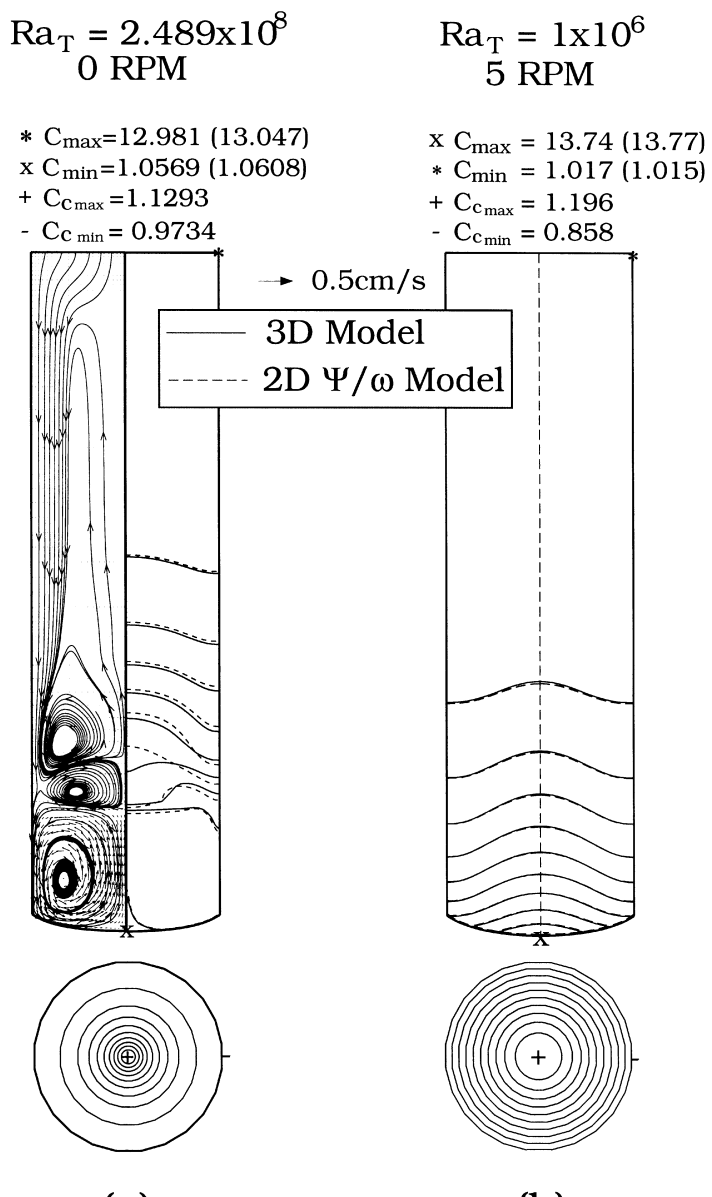

(a)

(b)

Fig. 2. Comparison of calculated results by $3 \mathrm{D}$ FVM and $2 \mathrm{D}$ $\Psi-\omega$ method [11] for (a) $\mathrm{Ra}_{\mathrm{T}}=2.489 \times 10^{8}(0 \mathrm{RPM}$ or $\mathrm{Ta}=0)$; (b) $\mathrm{Ra}_{\mathrm{T}}=1 \times 10^{6}\left(5 \mathrm{RPM}\right.$ or Ta $\left.=1.0139 \times 10^{4}\right)$.

\subsection{Ampoule tilting}

As mentioned previously, a perfect ampoule alignment with the gravity may not be possible in practice. During crystal growth, a small tilt (less than $2^{\circ}$ ) from the gravity orientation could be very common, and this may break axisymmetry $[5,6]$. Figs. $3 a$ and $b$ show the effect of $1.5^{\circ}$ tilt on the flow and the dopant segregation for $\mathrm{Ra}_{\mathrm{T}}=2.489 \times 10^{8}$. The same as before for $\mathrm{Ra}_{\mathrm{T}}=1 \times 10^{6}$, the tilt induces significant 3D flow and bulk dopant mixing; the convection due to the tilt becomes much stronger, 
which can be seen from the velocity fields in Fig. $3 \mathrm{~b}$. Accordingly, $C_{\min }$ is increased to 9.1484 (1.0569 in Fig. 3a). Furthermore, the radial segregation $\left(C c_{\max }-C c_{\min }\right)$ is reduced due to better local mixing. In fact, tilting the ampoule to enhance dopant mixing was also reported by Xiao et al. [6]. However, as shown in the dopant field at the interface in Fig. 3b, it is no longer axisymmetric. Although such a 3D segregation in the crystal can be further reduced in practice by solid-state diffusion. Still, it is in general not favored.

On the other hand, before the ampoule is tilted, if we apply $10 \mathrm{RPM}$ rotation $\left(\mathrm{Ta}=4.0556 \times 10^{4}\right)$ to the growth, as illustrated in Fig. 3c, the convection can be damped significantly and stretched axially as discussed by Lan before [11]. More importantly, the secondary cell disappears and the elongated main flow cell penetrates further into the bulk melt. As a result, the global mixing is enhanced by the $10 \mathrm{RPM}$ rotation even though the convection is significantly weaker. The radial segregation is reduced slightly, but this is not always true for ampoule rotation [11]. Again, tilting the ampoule $1.5^{\circ}$ induces 3D flows as shown in Fig. 3d. However, as compared with Fig. 3b, the convection is much weaker due to rotation. The bulk dopant mixing is reduced as well, which can be seen from the lower $C_{\text {min }}$ (6.876 versus 9.1484 in Fig. 3c) and the isoconcentration lines. In addition, due to rotation, the dopant field at the interface is distorted along the azimuthal direction. Unfortunately, the radial segregation is significantly increased by rotation; $C_{\max }$ is increased to 14.13 as compared with 12.166 in Fig. $3 b$ and 12.519 in Fig. 3c. Such an opposite effect may be caused by the secondary flow cells near the growth interface. Clearly, from the results of Fig. 3, the 10 RPM ampoule rotation, beside damping the flow, does not improve the growth. Therefore, for further study, one may need to use a higher rotation speed or use it for weaker buoyancy convection. We have chosen the later case for discussion.

As the buoyancy convection is reduced, which can be achieved by reducing temperature difference $\Delta T$ or gravity $g$, the rotation becomes more significant. Figs. $4 \mathrm{a}$ and $\mathrm{b}$ are still the effects of $1.5^{\circ}$ tilt for 3D flows and dopant segregation, but now for $\mathrm{Ra}_{\mathrm{T}}=1 \times 10^{6}$. As discussed before by Liang and

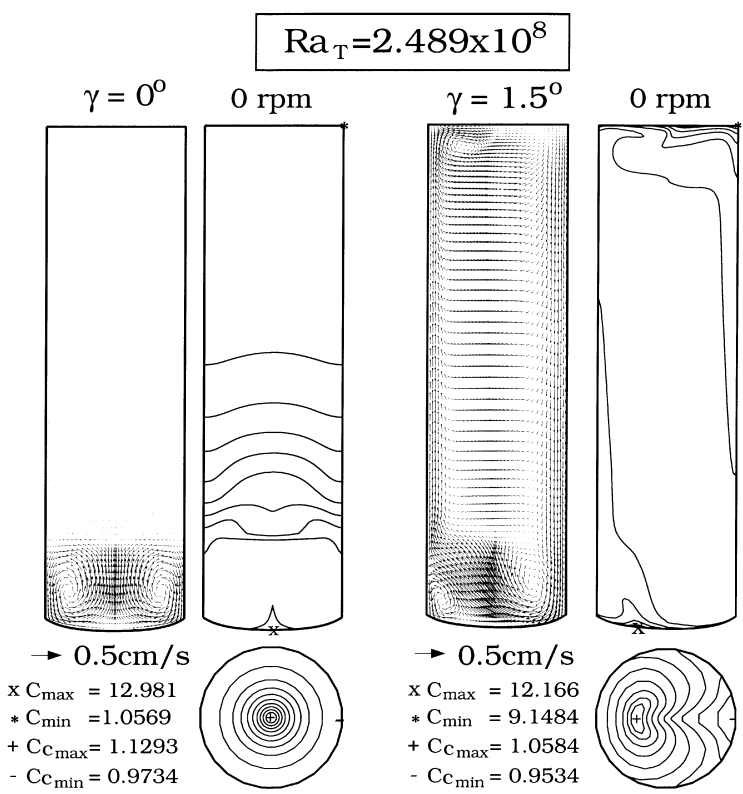

(a)

(b)

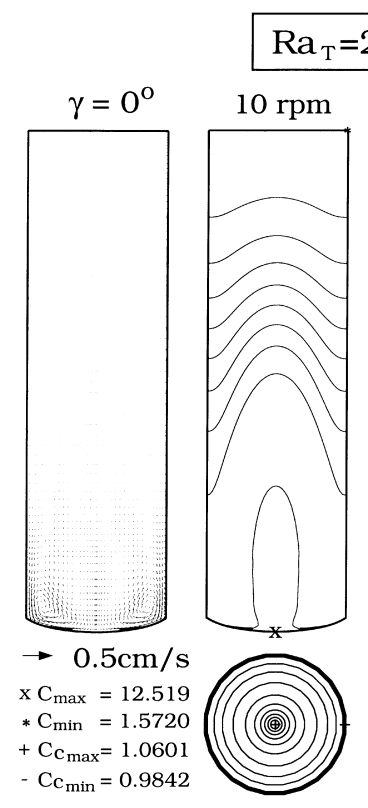

(c)

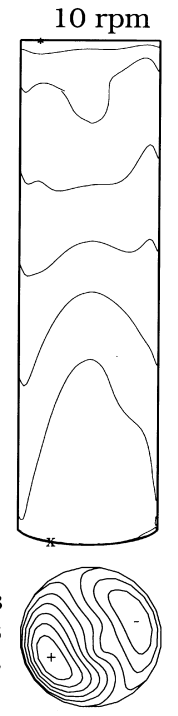

(d)
Fig. 3. Effects of ampoule tilting and rotation on the calculated flow patterns (LHS) and dopant fields (RHS) for $\mathrm{Ra}_{\mathrm{T}}=$ $2.489 \times 10^{8}$ : (a) $\gamma=0^{\circ}, \Omega=0 \mathrm{RPM} \quad(\mathrm{Ta}=0) ;\left(\right.$ b) $\gamma=1.5^{\circ}$, $\Omega=0 \mathrm{RPM}(\mathrm{Ta}=0) ;(\mathrm{c}) \gamma=0^{\circ}, \Omega=10 \mathrm{RPM}(\mathrm{Ta}=4.0556 \times$ $\left.10^{4}\right) ;\left(\right.$ d) $\gamma=1.5^{\circ}, \quad \Omega=10 \mathrm{RPM}\left(\mathrm{Ta}=4.0556 \times 10^{4}\right) ; \Delta C=$ $\left(C_{\max }-C_{\min }\right) / 10$. 
Lan [5], even for such a weak flow, the small tilt is significant. However, due to the poorer mixing, the radial segregation for both cases increases; $C_{\max }=14.13$ in a and 15.03 in $\mathrm{b}$. Interestingly, the 3D flow induced by the tilt increases the radial segregation even though the global mixing in enhanced. However, as 5 RPM ampoule rotation is introduced, the convection is damped significantly for both cases; we have purposely used a much smaller vector length scale to represent the velocity fields. The radial segregation in Fig. $4 c$ is reduced slightly by the rotation. Interestingly, although the convection in Fig. $4 d$ is still 3D, the dopant filed is nearly axisymmetric. Obviously, the diffusion predominates in the dopant segregation. As a result, as compared with Fig. 4b, the 3D effect of the ampoule tilting is significantly suppressed. The rotation pushes the segregation towards the diffusion-controlled regime and thus reduces the effect of ampoule tilting.

The results in Fig. 4 also implies that the rotation can be quite effective in reduced gravity, which in turn indicates that the faster rotation is needed for normal-gravity growth. However, during crystal growth in space, the gravity orientation is quite arbitrary and maybe changing with time due to orbiting or astronaut maneuver. Therefore, the situation is somewhat different from that due to the small tilt. To illustrate that, we further reduce $\mathrm{Ra}_{T}$ to $10^{4}$, and the effect of gravity orientation is shown in Fig. 5. As shown in Fig. 5a, if the gravity is the same as the growth orientation, the dopant field is almost the same with that for $\mathrm{Ra}_{\mathrm{T}}=0$, i.e., diffusion-controlled limit (more precisely, the convection contribution is only by the ampoule translation). Its velocity field (now shown here) is almost due to the ampoule translation only. However, as the gravity orientation is $45^{\circ}$ from the growth direction, significant $3 \mathrm{D}$ flow is induced. One may compare the velocity magnitude with that in Fig. 4a $\left(\mathrm{Ra}_{\mathrm{T}}=10^{6}, \gamma=0^{\circ}\right.$, and $\left.0 \mathrm{RPM}\right)$ and find that their convection levels are comparable. Accordingly, the radial segregation is thus severe being about the same order as that in Fig. 4a. Further increasing the gravity orientation to $90^{\circ}$ does not change much the convection and the segregation (Fig. 5c). Interestingly, the result for $135^{\circ}$ in Fig. 5d is the same as that for $45^{\circ}$. This is because the radial

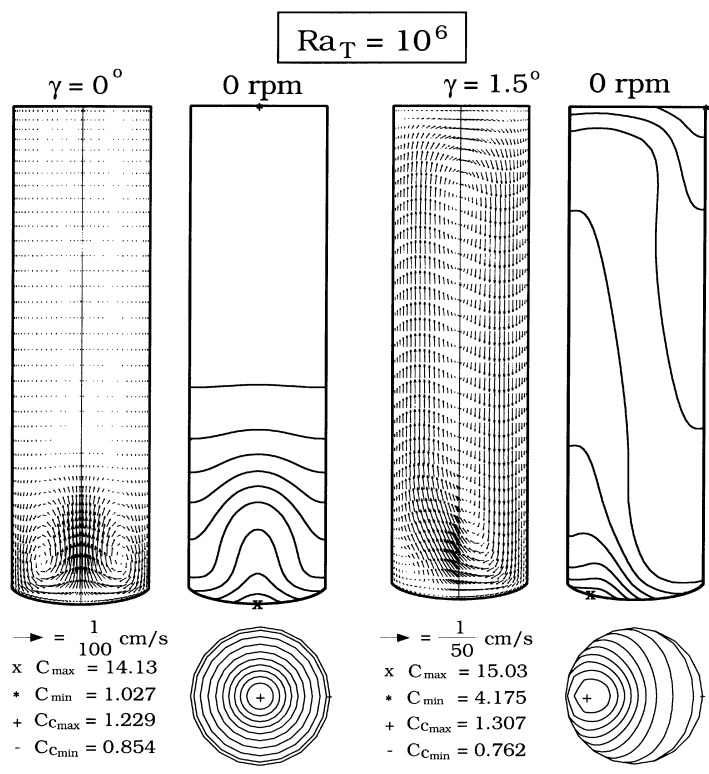

(a)

(b)

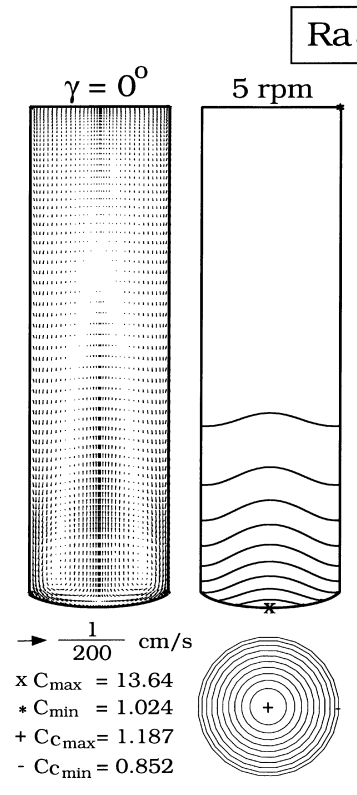

(c)
)
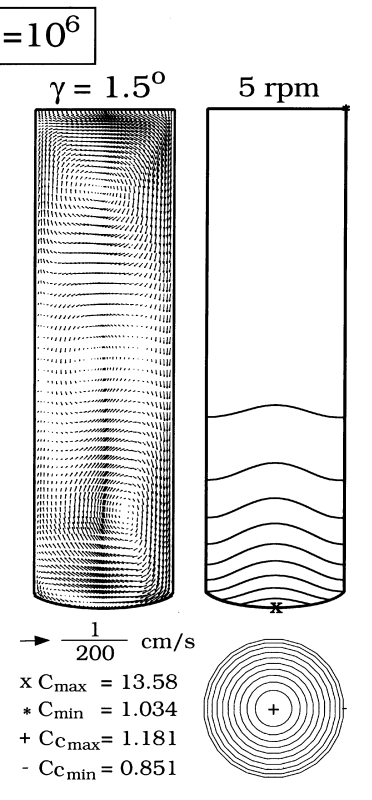

(d)

Fig. 4. Effects of ampoule tilting and rotation on the calculated flow patterns (LHS) and dopant fields (RHS) for $\mathrm{Ra}_{\mathrm{T}}=1 \times 10^{6}$; (a) $\gamma=0^{\circ}, \Omega=0 \mathrm{RPM}(\mathrm{Ta}=0)$; (b) $\gamma=1.5^{\circ}$, $\Omega=0 \mathrm{RPM} \quad(\mathrm{Ta}=0) ; \quad(\mathrm{c}) \gamma=0^{\circ}, \quad \Omega=5 \mathrm{RPM} \quad(\mathrm{Ta}=$ $\left.1.0139 \times 10^{4}\right) ;(\mathrm{d}) \gamma=1.5^{\circ}, \Omega=5 \mathrm{RPM}\left(\mathrm{Ta}=1.0139 \times 10^{4}\right) ; \Delta C=$ $\left(C_{\max }-C_{\min }\right) / 10$. 


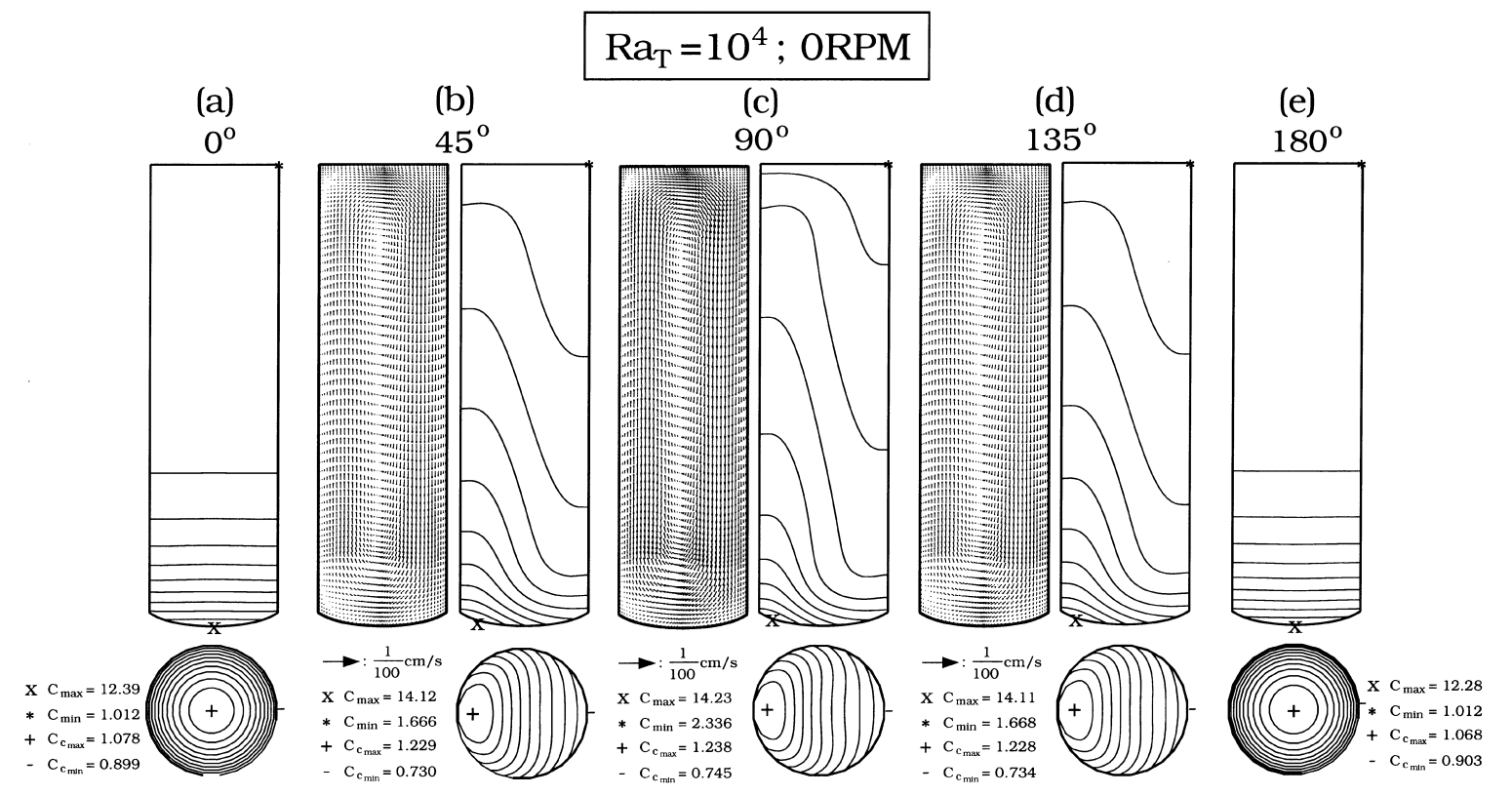

Fig. 5. Effects of gravity orientation on the calculated flow patterns (LHS) and dopant fields (RHS) for $\operatorname{Ra}_{\mathrm{T}}=1 \times 10^{4}$ : (a) $\gamma=0^{\circ}$; (b) $\gamma=45^{\circ}$; (c) $\gamma=90^{\circ}$; (d) $\gamma=135^{\circ}$; (e) $\gamma=180^{\circ}$.

temperature differences for convection in both cases are the same. Due to the same reason, the results for the inverted growth in Fig. 5e are almost the same as that in Fig. 5a.

The 3D flows induced in Figs. $5 \mathrm{~b}$ and $\mathrm{c}$ are similar to the 2D results by Arnold et al. [22] in a microgravity condition. The feasibility of using magnetic fields to suppress the induced convection was investigated by Yao et al. [9] for the growth of $\mathrm{PbSnTe}$, and more than $30 \mathrm{kG}$ of magnetic field was found necessary to achieve a nearly diffusion-controlled segregation. In fact, using steady ampoule rotation, which is much simpler, can be effective as well. As shown in Fig. 6, the 5 RPM steady ampoule rotation can almost eliminate the gravitational effect. The dopant fields imply that the diffusion-controlled limit is achieved. Although the use of ampoule rotation to damp 3D flows has not been adopted in space experiments, it is believed that it may be an effective way for better growth control. In fact, a recent study by Polovko et al. [23] pointed out that the Coriolis force due to spacecraft rotation has significant influences on the thermal convection and thus the segregation; a cubic fluid system was investigated. Indeed, steady ampoule rotation could be a much easier way for growth control. On the other hand, we have assumed that the heating is perfectly axisymmetric here. However, if the heating is not uniform, timedependent flows and growth striations can be introduced by the rotation, which makes the growth even worse. Therefore, a feasible way to minimize such an effect is to rotate the furnace and the ampoule together.

\subsection{Nonuniform heating}

In addition to the ampoule tilting or gravity orientation, asymmetric heating can also induce 3D flow as illustrated by Liang and Lan [5]. As shown in Fig. $7 \mathrm{a}$, even the nonuniformity is only $2^{\circ} \mathrm{C}$ $\left(\Delta T_{\mathrm{dp}}=1^{\circ} \mathrm{C}\right)$, significant 3D flow can be induced; the total temperature difference $\Delta T=350^{\circ} \mathrm{C}$. Unlike the tilting effect, the $3 \mathrm{D}$ flow is more localized to the growth interface, where the heating nonuniformity is imposed. Meanwhile, due to the 


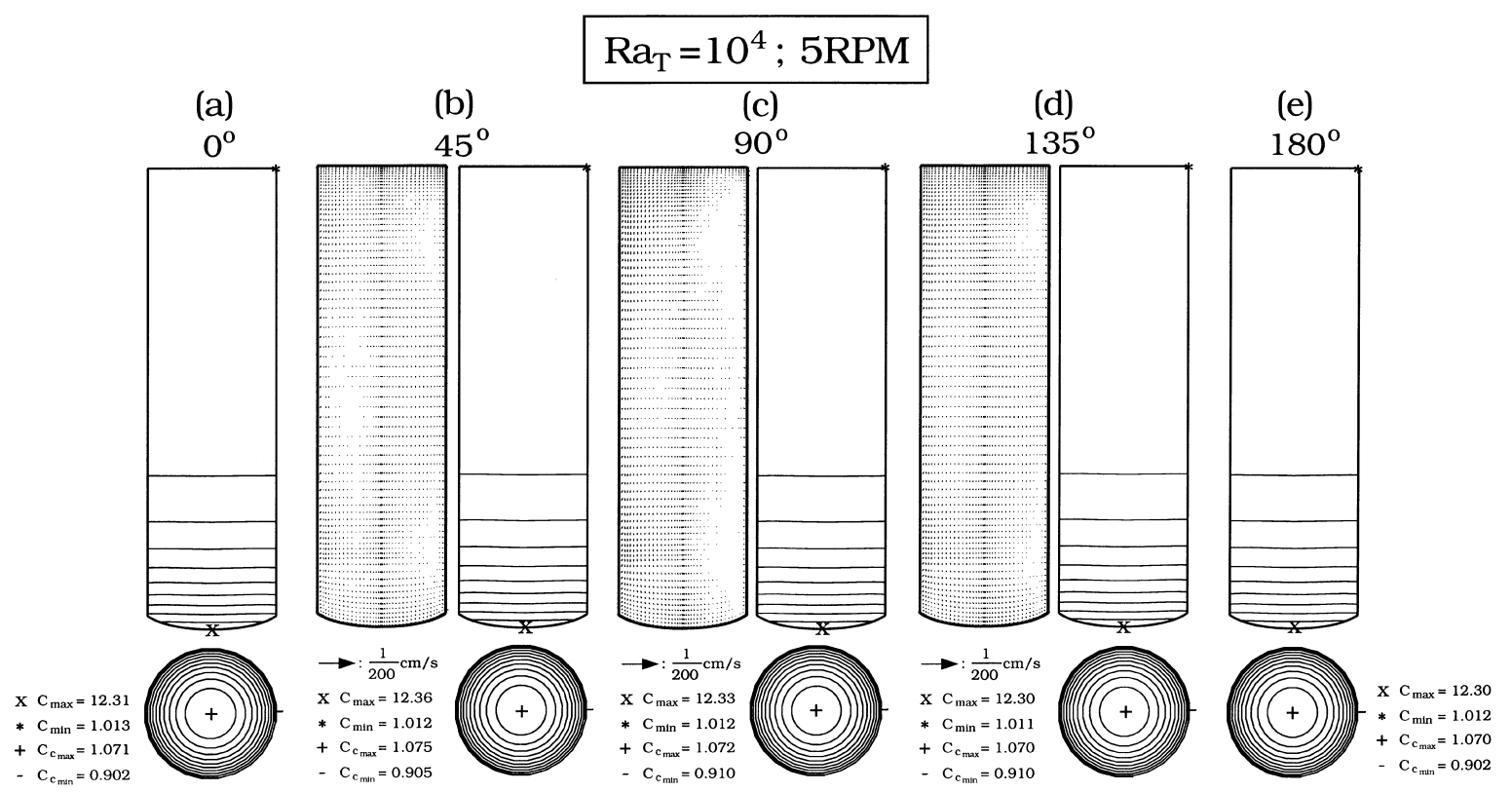

Fig. 6. Effects of $5 \mathrm{RPM}$ rotation $\left(\mathrm{Ta}=1.0139 \times 10^{4}\right)$ on the calculated flow patterns $(\mathrm{LHS})$ and dopant fields $(\mathrm{RHS})$ for $\mathrm{Ra} \mathrm{T}_{\mathrm{T}}=1 \times 10^{4}$ at different gravity orientations: (a) $\gamma=0^{\circ}$; (b) $\gamma=45^{\circ}$; (c) $\gamma=90^{\circ}$; (d) $\gamma=135^{\circ}$; (e) $\gamma=180^{\circ}$.

better local dopant mixing, the radial segregation is reduced slightly. If one examines the interface shape carefully, the interface is no longer axisymmetric; the left side is lower due to the higher ambient temperature there. With 10 RPM rotation, the convection is damped significantly. Especially, as shown by the dopant field away from the interface, it becomes almost axisymmetric. However, severe abnormal dopant segregation is found near the growth interface; $C c_{\max }$ is increased to 2.0111 and $C c_{\min }$ is reduced to only 0.4425 . In fact, due to the asymmetric heating, the asymmetric interface is a problem. When the ampoule is rotating, continuous melting $\left(\boldsymbol{n} \cdot \boldsymbol{e}_{\phi}>0\right)$ and growth $\left(\boldsymbol{n} \cdot \boldsymbol{e}_{\phi}<0\right)$ proceed. As a result, the melting part leads to the lower dopant concentration due to the dilution from the crystal, while the growth part to a higher concentration. This can be further explained by the interface dopant balance in Eq. (10), where the rotation term becomes important when $\left(\boldsymbol{n} \cdot \boldsymbol{e}_{\phi}\right)$ is not trivial. As mentioned previously, this boundary condition is not exact, because the interface dopant equilibrium may not be achieved. However, even $(1-K)$ $C$ in Eq. (10) is replaced by $C-C_{0}$, the difference is still not much. Therefore, such a severe rotational segregation is believed to be realistic.

Even without buoyancy convection, the rotational segregation is still significant. Fig. 8 shows the effects of $5 \mathrm{RPM}$ rotation for the asymmetric heating at $\operatorname{Ra}_{\mathrm{T}}=0$ and $10^{6}$. At $\operatorname{Ra}_{\mathrm{T}}=0$ and $\Omega=0$, the asymmetric segregation in Fig. $8 \mathrm{a}$ is due to the asymmetric interface shape. As the ampoule is rotated at 5 RPM, as shown in Fig. 8b, the dopant field near the interface is affected significantly due to the rotational segregation at the interface, and the effect increases with the increasing radial coordinate due to the increasing azimuthal velocity $(r \Omega)$. With the buoyancy convection at $\mathrm{Ra}_{\mathrm{T}}=1 \times 10^{6}$ (Fig. 8c), the rotational segregation is further enhanced. The dopant field is further distorted by the convection in the rotational direction. Away from the interface, the dopant field is still nearly axisymmetric. One may easily imagine that the rotational segregation is also the cause of the rotation striations due to the continuous growth and remelting. The rotational striations in Czochralski growth have been known for a long time [17], but to our best knowledge no numerical work has been 


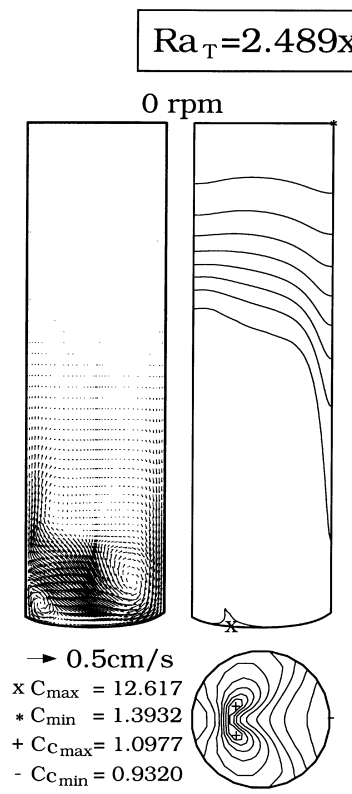

(a)

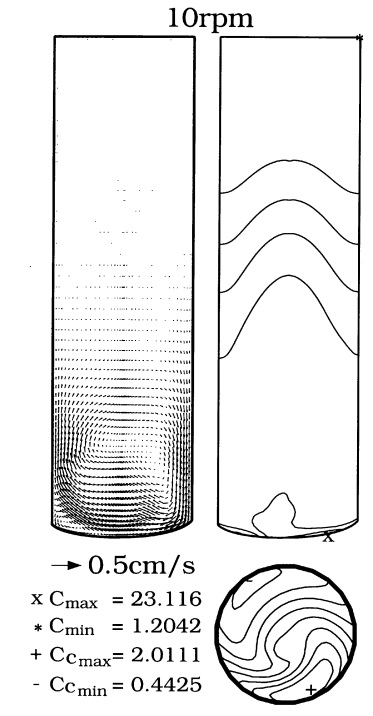

(b)
Fig. 7. Effects of asymmetric heating and ampoule rotation on the calculated flow patterns (LHS) and dopant fields (RHS) for $\mathrm{Ra}_{\mathrm{T}}=2.489 \times 10^{8}$ : (a) $\Delta T_{\mathrm{dp}}=1^{\circ} \mathrm{C}, \Omega=0 \mathrm{RPM}(\mathrm{Ta}=0)$; (b) $\Delta T_{\mathrm{dp}}=1^{\circ} \mathrm{C}, \Omega=10 \mathrm{RPM}\left(\mathrm{Ta}=4.0556 \times 10^{4}\right)$.

reported for that. For VB growth, the numerical simulation of the rotational segregation is conducted here for the first time. By considering the axial growth and crystal rotation, an outcome from this effect is the rotational striations proposed by Gatos [17]. Furthermore, from Fig. 8, it is clear that even for only 5 RPM rotation, the continuous growth and melting rate may be much higher than the axial growth rate $\left(U_{\text {amp }}\right)$ to induce the severe segregation. Therefore, in such a case, rotation is believed to be harmful. Constitutional supercooling due to rotation can be an important issue as well.

\section{Conclusions}

The effects of steady ampoule rotation on the 3D flows and dopant segregation induced by ampoule tilting or asymmetric heating in Bridgman crystal growth are investigated numerically for the first time. It is found that rotation can damp the $3 \mathrm{D}$

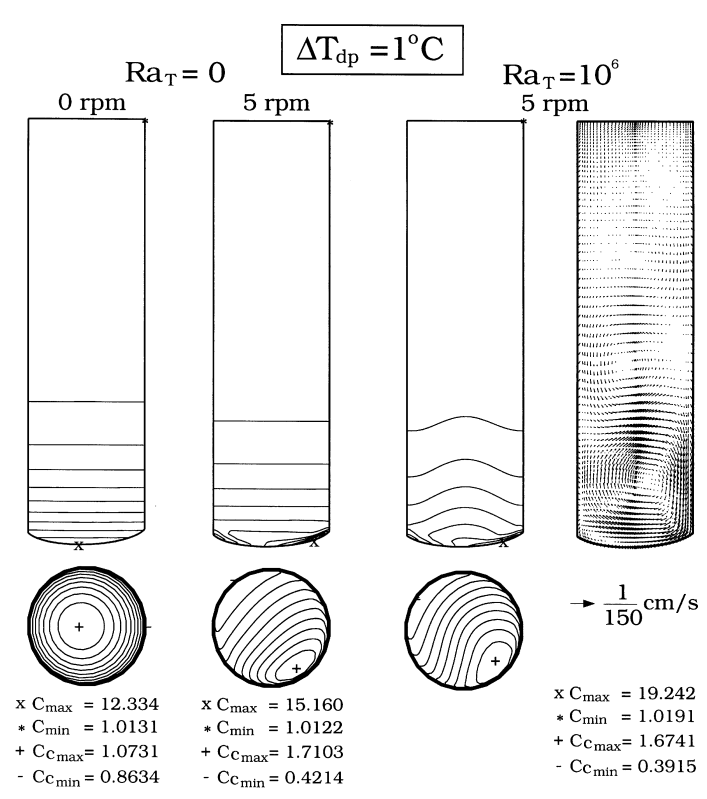

(a)

(b)

(c)

Fig. 8. Effects of 5 RPM ampoule rotation on dopant fields for $\Delta T_{\mathrm{dp}}=1{ }^{\circ} \mathrm{C}$ : (a) $\mathrm{Ra}_{\mathrm{T}}=0, \Omega=0 \mathrm{RPM}$; (b) $\mathrm{Ra}_{\mathrm{T}}=0, \Omega=$ 5 RPM; (c) $\mathrm{Ra}_{\mathrm{T}}=1 \times 10^{6}, \Omega=5 \mathrm{RPM}$.

flows; however, it is still hard to achieve an axisymmetric convection. Nevertheless, if the convection can be suppressed sufficiently, the diffusion becomes dominant in the segregation. We still have a good chance to have a nearly axisymmetric growth. This is particularly true for the growth in a reduced-gravity environment using low-speed rotation, even through the gravity orientation is arbitrary.

The effects of ampoule rotation for asymmetric heating are more complicated. In addition to the 3D flows, due to the asymmetric growth interface, the continuous melting and growth can lead to severe radial segregation. This rotational segregation is still significant even at low-speed rotation and without thermal convection. Although the present simulation uses a pseudo-steady-state approximation, which may not describe accurately the dopant field, the calculated results here may still provide a useful insight to the detailed transport phenomena in Bridgman crystal growth. Again, 
further crystal growth experiments are necessary to verify the results proposed here.

\section{Acknowledgements}

The authors are grateful for the support from the National Science Council and the National Center for High Performance Computing of the Republic of China under Grant No. 88-2214-E-002-006.

\section{References}

[1] W.A. Gault, E.M. Monberg, J.E. Clemans, J. Crystal Growth 74 (1986) 491.

[2] K. Hoshikawa, H. Nakanishi, H. Kohda, M. Sasaura, J. Crystal Growth 94 (1989) 643.

[3] E.M. Monberg, W.A. Gault, F. Simchock, F. Domingguez, J. Crystal Growth 83 (1987) 174

[4] E.M. Monberg, in: D.T.J. Hurle (Ed.), Handbook of Crystal Growth 2a: Basic Techniques, North-Holland, Amsterdam, 1994, p. 52.

[5] M.C. Liang, C.W. Lan, J. Crystal Growth 167 (1996) 320.
[6] Q. Xiao, S. Kuppurao, A. Yeckel, J.J. Derby, J. Crystal Growth 167 (1996) 292.

[7] D.H. Kim, P.M. Adornato, R.A. Brown, J. Crystal Growth 89 (1988) 339.

[8] S. Motakef, J. Crystal Growth 104 (1990) 833.

[9] M. Yao, A. Chait, A.L. Fripp, W.J. Debnam, J. Crystal Growth 173 (1997) 467.

[10] V. Uspenskii, J.J. Favier, Int. J. Heat Mass Transfer 37 (1994) 691.

[11] C.W. Lan, J. Crystal Growth 197 (1999) 983.

[12] C.W. Lan, J.H. Chian, J. Crystal Growth 203 (1999) 286.

[13] A. Yeckel, F.P. Doty, J.J. Derby, J. Crystal Growth 203 (1999) 87.

[14] C.W. Lan, J. Crystal Growth, in preparation.

[15] W.A. Arnold, W.R. Wilcox, F. Carlson, A. Chait, L.L. Regel, J. Crystal Growth 119 (1992) 24.

[16] C.W. Lan, M.C. Liang, J. Crystal Growth 186 (1998) 203.

[17] H.C. Gatos, J. Electrochem. Soc. 122 (1975) 287.

[18] M.C. Liang, C.W. Lan, J. Comp. Phys. 127 (1996) 330.

[19] C.W. Lan, M.C. Liang, J. Comp. Phys. 152 (1999) 55.

[20] P.M. Adornato, R.A. Brown, J. Crystal Growth 80 (1987) 155.

[21] C.W. Lan, F.C. Chen, Comput. Methods Appl. Mech. Engng. 131 (1996) 191.

[22] W.A. Arnold, D.A. Jacqmin, R.L. Gaug, A. Chait, Spacecraft Rockets 28 (1991) 238.

[23] A.Y. Polovko, V.V. Sazonov, V.S. Yuferev, J. Crystal Growth 198 (1999) 182. 